\title{
Anti-infective ophthalmic preparations in general practice
}

\author{
Smit D, MBChB, Dip Ophth(SA), FC Ophth(SA), MMed(Ophth)(Stell) \\ Department of Ophthalmology Faculty of Health Sciences, Stellenbosch University, Tygerberg Hospital \\ Correspondence to: Derrick Smit, e-mail: dpsmit@sun.ac.za \\ Keywords: anti-infective ophthalmic preparations, general practice
}

\begin{abstract}
Ocular infections may be bacterial, viral, fungal or parasitic in aetiology. Pharmacological preparations are available to treat infections that are caused by these groups of organisms. The majority of these preparations are intended for topical administration, although some systemically administered agents may be needed to treat or prevent specific ocular infections. This article discusses the different anti-infective options that are available to general practitioners to treat infections caused by each aetiological group. It also discusses the role that is played by povidone-iodine and antibiotic-steroid combinations to manage eye infections. A summary of all these drugs is provided in table form for easy reference.
\end{abstract}

C) Medpharm

S Afr Fam Pract 2012;54(4):302-307

\section{Introduction}

Ocular infections are caused by a wide variety of infectious agents and present in many different guises. The causative agents may broadly be divided into the same four groups of organisms that cause infection elsewhere in the body: viral, bacterial, fungal and parasitic. All of these groups are capable of infecting the superficial layers of the eye, such as the conjunctiva and the cornea, as well as deeper ocular structures, such as the vitreous humour and the retina. The route of administration of the appropriate anti-infective preparation is largely determined by the site of the primary infection. Therefore, cases of infectious conjunctivitis and keratitis are primarily treated with topical preparations, whereas intraocular infections, such as endophthalmitis or retinitis, usually require periocular, intraocular or systemic administration of anti-infective preparations.

\section{Classification of anti-infective ophthalmic preparations}

Preparations are available to treat eye infections that are caused by organisms in all four abovementioned groups. Table I provides an outline of the different drug classes, as well as the specific available drugs in each class. A fifth class is also included. This contains preparations that are effective against more than one class of infectious organisms.

\section{Antibacterial ophthalmic preparations}

\section{Sulphacetamide}

Available preparation: $10 \%$ ointment
Sulphacetamide is a bacteriostatic sulphonamide with limited ophthalmic use. It may be combined with oral erythromycin, azithromycin or clarithromycin for the treatment of chlamydial conjunctivitis, and should then be used two or three times a day for 3-6 weeks. ${ }^{1}$ It should not be used by patients with a known hypersensitivity to sulphonamide drugs. ${ }^{2}$

\section{Propamidine isethionate}

Available preparation: $1 \mathrm{mg} / \mathrm{ml}$ drops

Propamidine isethionate may be used for treating mild bacterial conjunctivitis caused by staphylococci and streptococci, but is not effective against $P$ seudomonas spp. or Escherichia coli. If a superficial eye infection does not improve within one week of treatment, a change in therapy to a drug with a broader spectrum should be considered. Propamidine isethionate also plays an important role in the treatment of Acanthamoeba keratitis, which needs to be excluded in contact lens wearers who present with a corneal infection that initially mimics herpetic keratitis, but does not respond to the usual treatment for that condition.

\section{Fusidic acid}

\section{Available preparation: $10 \mathrm{mg} / \mathrm{g}$ viscous drops}

Generally, fusidic acid is considered to have a narrow spectrum of activity which predominantly covers staphylococcal infections such as blepharitis or conjunctivitis. However, in a Canadian study that compared the topical use of $1 \%$ fusidic acid to $0.3 \%$ tobramycin to treat acute bacterial conjunctivitis, no difference in efficacy was demonstrated between the two drugs. The twice-daily dosage regimen was also found to be more convenient than the frequent installations required for tobramycin drops. ${ }^{3}$ 


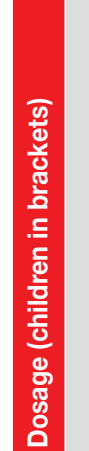

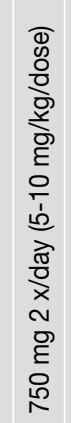

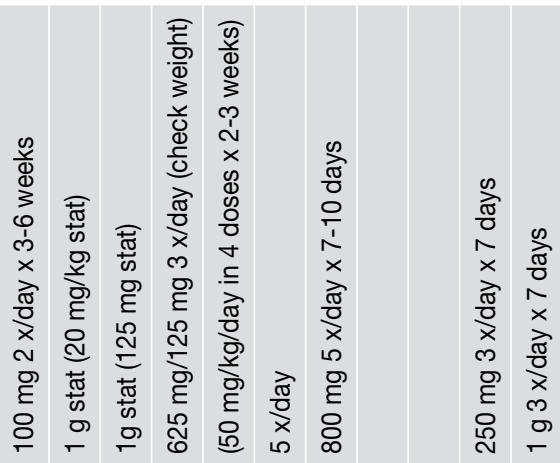

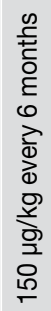

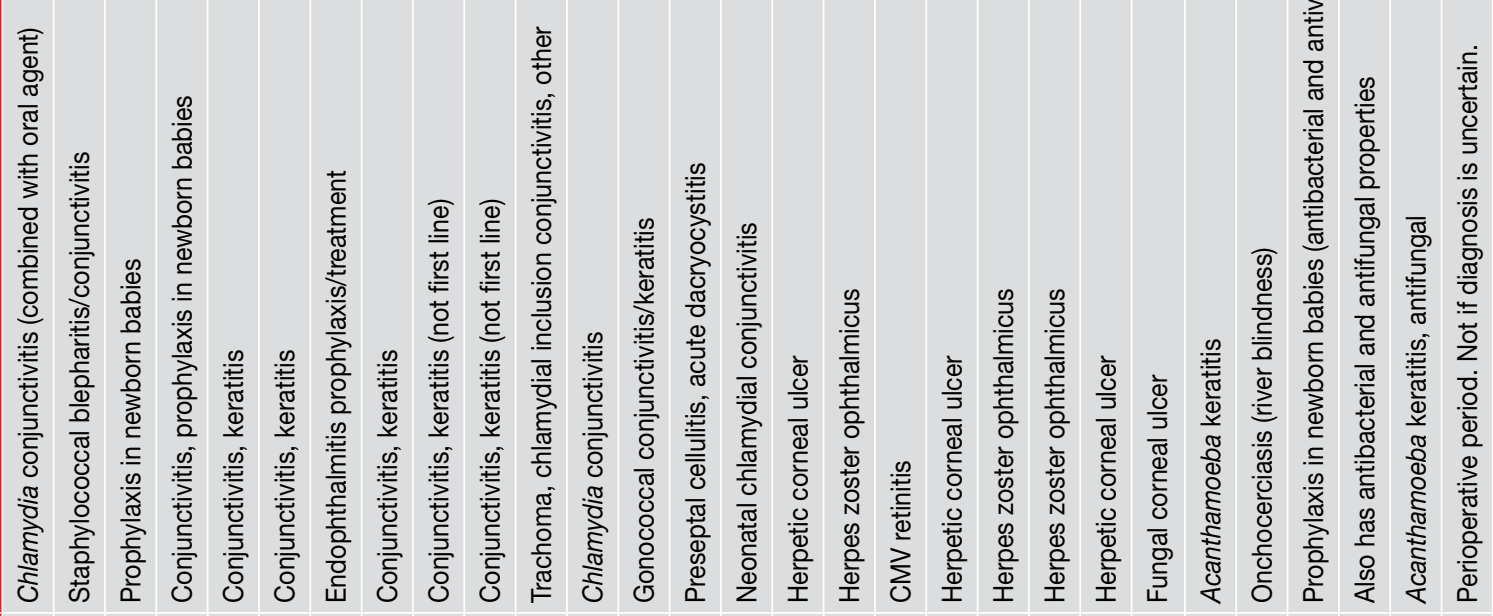

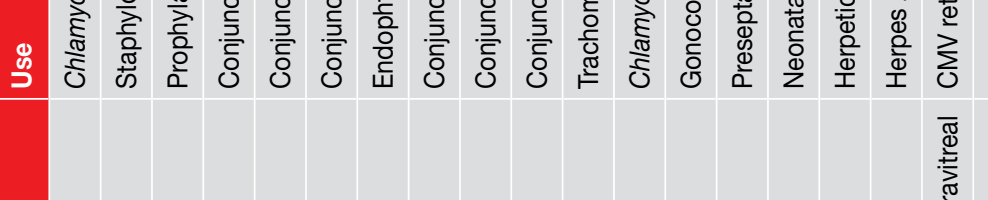

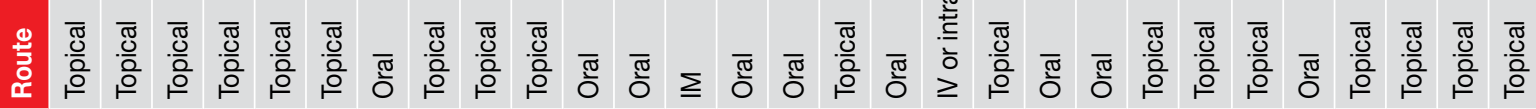

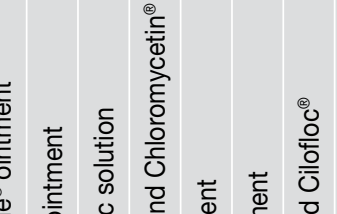

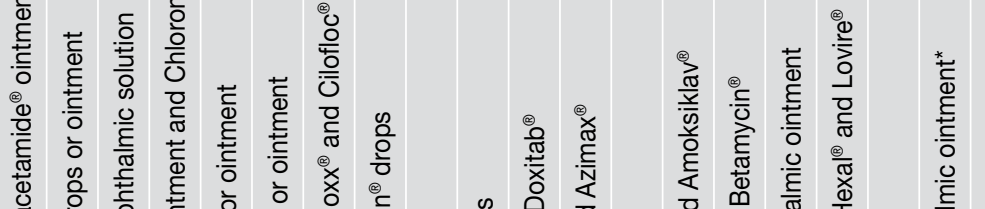

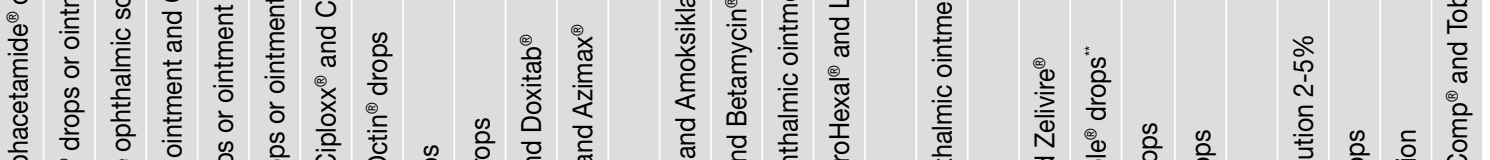

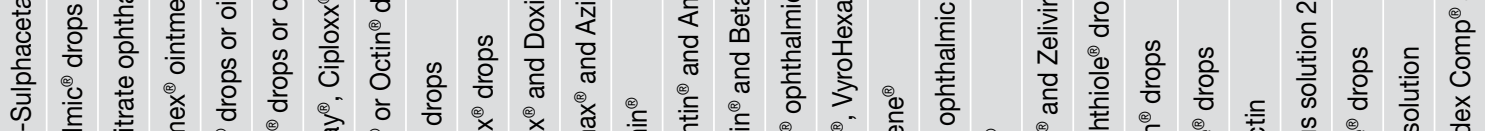

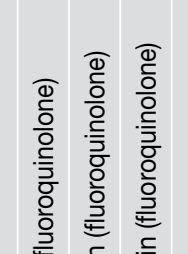

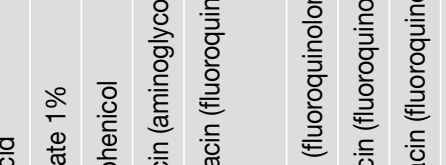

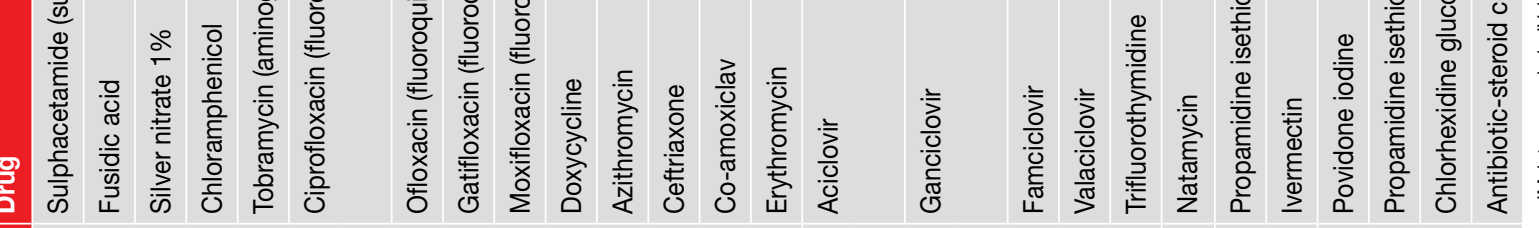

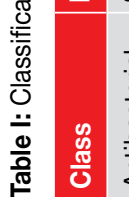

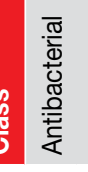

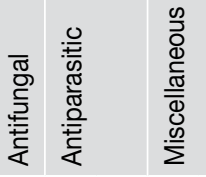




\section{Silver nitrate}

The story of Dr Carl Credé and the prevention of ophthalmia neonatorum with silver nitrate drops is well worth reading, and may be accessed free of charge at the following web address: http://www.ncbi.nlm.nih.gov/pmc/articles/ PMC1721147/pdf/v083p0F158.pdf

Very few interventions have had such a profound impact on a medical condition worldwide. ${ }^{4}$ Credé originally advocated the use of a $2 \%$ silver nitrate solution, but this concentration was later halved. Today, silver nitrate is no longer widely used, although it should still be considered in cases in which Neisseria gonorrhoeae infection is prevalent. However, it does not provide adequate protection against Chlamydia trachomatis infection. This, combined with the occurrence of a mild chemical conjunctivitis in neonates, is the main reason why its use has been discontinued.

\section{Chloramphenicol}

Available preparations: $0.5 \%$ preserved drops, $1 \%$ ointment and $0.5 \%$ preservative-free, single-dose units

Chloramphenicol is the most frequently used broadspectrum topical antibiotic in South Africa and is effective in more than $99 \%$ of superficial bacterial eye infections. ${ }^{2}$ It is clinically effective against the majority of Gram-positive and Gram-negative organisms. Therefore, it still plays an integral role in the first-line treatment of bacterial conjunctivitis and blepharitis.

The ocular toxicity of topical chloramphenicol is low, although it may serve as a trigger for delayed allergic contact dermatitis or conjunctivitis. Aplastic anaemia and other blood dyscrasias have been linked to the ocular use of chloramphenicol, although it has been shown that the risk is less than one per million treatment courses. Therefore, use of this drug to treat superficial eye infections should not be precluded. ${ }^{5}$ In South Africa, it is also recommended for the prevention of neonatal conjunctivitis. ${ }^{2}$ Interestingly enough, the only drug that has been approved by the United States Food and Drug Administration for the prevention of neonatal conjunctivitis is erythromycin $0.5 \%$ ophthalmic ointment. This is not available in South Africa. ${ }^{6}$

\section{Tobramycin}

Available preparations: $3 \mathrm{mg} / \mathrm{ml}$ drops and $3 \mathrm{mg} / \mathrm{g}$ ointment

Since gentamycin eye drops were discontinued a few years ago, tobramycin is now the only commercially available topical aminoglycoside preparation in South Africa. It is active against most Gram-negative bacteria, as well as a few aerobic Gram-positive microbes, especially staphylococci. It has been a reliable treatment option for superficial eye infections for many years, and was previously often used as the gold standard in clinical trials in which newer preparations needed to be compared to a well-established drug.
It may cause transient stinging after installation and hypersensitivity reactions have been reported, but these are rare.

\section{Ciprofloxacin}

Available preparations: $3 \mathrm{mg} / \mathrm{ml}$ drops and $3 \mathrm{mg} / \mathrm{g}$ ointment Ciprofloxacin, a class II second-generation fluoroquinolone, has a broad spectrum of activity against both Grampositive and Gram-negative bacteria, although it is often not effective against Streptococcus pneumoniae. However, it does have more activity against $P$ seudomonas aeruginosa than any other fluoroquinolone that is currently available. ${ }^{7}$ It is effective is most cases of bacterial conjunctivitis and will also successfully treat the majority of small peripheral corneal ulcers. Oral administration of ciprofloxacin results in high intraocular levels of the drug. Therefore, it is recommended as prophylaxis for traumatic endophthalmitis in all open-eye injuries. This includes paediatric cases since the benefit is considered to outweigh the risk of damage to cartilage in weight-bearing joints. The recommended dose in adults is $750 \mathrm{mg}$ twice daily, and in children, $5-10 \mathrm{mg} / \mathrm{kg} /$ dose twice daily, and up to a maximum of $500 \mathrm{mg}$ per dose. ${ }^{2}$

\section{Ofloxacin}

Available preparations: $3 \mathrm{mg} / \mathrm{ml}$ drops and $3 \mathrm{mg} / \mathrm{g}$ ointment Ofloxacin is another class II second-generation fluoroquinolone. Therefore, it has a similar spectrum of activity and application to ciprofloxacin, although it is not as effective against $P$. aeruginosa.

\section{Gatifloxacin}

Available preparation: $3 \mathrm{mg} / \mathrm{ml}$ drops

Gatifloxacin is a third-generation fluoroquinolone with a Gram-negative spectrum that is similar to that of the second-generation drugs, but with improved Gram-positive coverage. It is two to four times more active against S. pneumoniae than levofloxacin, another third-generation fluoroquinolone. ${ }^{7}$ Preferably, gatifloxacin should not be used as a first-line drug. It should rather be reserved to treat cases that are unresponsive to more conventional drugs. It is registered for the treatment of bacterial conjunctivitis that is caused by staphylococci, streptococci, Haemophilus influenzae and Corynebacterium propinquum. It is also very effective in treating more severe cases of bacterial keratitis. ${ }^{2}$

\section{Moxifloxacin}

\section{Available preparation: $5 \mathrm{mg} / \mathrm{ml}$ drops}

Moxifloxacin is another third-generation fluoroquinolone that covers the same spectrum as gatifloxacin, but demonstrates superior activity to $S$. pneumoniae. ${ }^{7}$ It is also effective against Acinetobacter spp., E. coli, Serratia marcescens and Chlamydia trachomatis. Despite only being licenced to treat bacterial conjunctivitis, moxifloxacin is often highly effective in treating moderate to severe bacterial keratitis. Therefore, ideally it should be reserved for more complicated cases to minimise the emergence of antibiotic resistance. 


\section{Doxycycline}

Tetracycline eye ointment is indicated for the treatment of chlamydial inclusion conjunctivitis. Unfortunately, it is not available in South Africa. ${ }^{8}$ An alternative treatment option for this condition is an oral tetracycline, such as doxycycline $100 \mathrm{mg}$ twice daily for three weeks. Trachoma, another chlamydial infection, may also be treated with the same oral dose of doxycycline, but requires a longer treatment period of 3-6 weeks. Furthermore, oral doxycycline is used in the treatment of other eye conditions, such as posterior blepharitis and ocular rosacea, but the mechanism of action is not antibacterial in these conditions.

\section{Azithromycin}

Azithromycin provides a further treatment option for chlamydial inclusion conjunctivitis. In adults, a single dose of $1 \mathrm{~g}$ is given orally, while a single dose of $20 \mathrm{mg} / \mathrm{kg},{ }^{1}$ or alternatively three daily doses of $10 \mathrm{mg} / \mathrm{kg}$, may be administered to neonates. ${ }^{2}$ Chlamydial conjunctivitis is a sexually transmitted infection in adults. Therefore, appropriate steps need to be taken to trace and treat all sexual partners.

\section{Ceftriaxone}

Ceftriaxone is a third-generation cephalosporin with excellent action against organisms such as $H$. influenzae, S. pneumoniae and $\mathrm{N}$. gonorrhoeae. ${ }^{2}$ Therefore, it is used to treat gonococcal conjunctivitis in both adults and neonates. A single dose of $1 \mathrm{~g}$ should be given intramuscularly to adults, while a single dose of $125 \mathrm{mg}$ should be given to neonates. If corneal involvement is present, a topical fluoroquinolone or tobramycin for use every hour should also be commenced before the patient is referred to an ophthalmologist. ${ }^{1}$ Once again, the sexual partners of infected adults should be traced and treated.

\section{Co-amoxiclav}

Co-amoxiclav is often used to treat soft tissue infections around the eye, since it not only provides good Gram-positive and Gram-negative cover, but also exhibits good efficacy against anaerobic organisms. Mild cases of dacryocystitis and preseptal cellulitis in patients who are otherwise well may safely be treated with co-amoxiclav in a family practice setting. Patients who are acutely ill, or those who may require surgical incision and drainage, should preferably be hospitalised and referred for an ophthalmology opinion.

\section{Erythromycin}

Erythromycin is a macrolide antibiotic that is effective against neonatal chlamydial conjunctivitis. The recommended oral dosage is $50 \mathrm{mg} / \mathrm{kg} /$ day in four divided doses for 2-3 weeks. This should be combined with topical sulphacetamide to be applied two to three times a day for 3-6 weeks, since neither erythromycin nor tetracycline are available in a topical preparation in South Africa.

\section{Antiviral ophthalmic preparations}

\section{Aciclovir}

Aciclovir is a guanosine analogue that is effective against a variety of herpesviridae, including herpes simplex 1 and 2, varicella-zoster virus and Epstein Barr virus. Aciclovir shows a high degree of selectivity for herpes-virus-infected cells, as a result of its unique mechanism of action. In short, phosphorylation of aciclovir to aciclovir monophosphate occurs more efficiently in virus-infected cells, because of the presence of viral thymidine kinase. The aciclovir monophosphate is then converted to a triphosphate form which inhibits viral DNA polymerase to a much greater extent than host cell DNA polymerase. Therefore, aciclovir has little effect on DNA synthesis in normal cells, but is a potent inhibitor of herpes-virus replication. ${ }^{9}$

Aciclovir $3 \%$ ointment is the drug of choice when treating herpetic conjunctivitis and dendritic corneal ulcers. It should be applied five times per day. Referral to an ophthalmologist may be necessary if a dendritic ulcer has not healed after seven days of treatment. If topical acyclovir is not available, oral acyclovir $400 \mathrm{mg}$ five times per day may be used as an alternative since orally administered aciclovir reaches therapeutic levels in the precorneal tear film. Oral aciclovir is more effective than a topical application in treating vesicular eruptions of the periocular skin in primary herpetic blepharoconjunctivitis. In a general practice setting, oral aciclovir is the most important drug in the treatment of herpes zoster ophthalmicus. The recommended dosage in adults is $800 \mathrm{mg}$ five times a day for $7-10$ days, but this dosage should be reduced in patients with renal impairment.

\section{Ganciclovir}

Ganciclovir is another guanosine analogue that is effective against all the herpesviridae, but is 10-25 times more active against cytomegalovirus (CMV) due to its specific chemical structure. ${ }^{9}$ Recently, a topical $0.15 \%$ preparation was launched in the USA to treat herpes keratitis. It was shown to be as effective as topical aciclovir for this indication and will hopefully be available in South Africa soon. Ganciclovir is also used by ophthalmologists to treat $\mathrm{CMV}$ retinitis in immunocompromised patients. It may be administered intravenously or by injection into the vitreous humour.

\section{Famciclovir}

Famciclovir is a prodrug of penciclovir and has a similar mechanism of action to aciclovir, in that it inhibits viral DNA polymerase. It is well absorbed orally and requires a lower dose and less frequent administration than aciclovir in the treatment of herpes zoster ophthalmicus. Unfortunately, it is considerably more expensive than aciclovir. The recommended dosage is $250 \mathrm{mg}$ three times a day for seven days. 


\section{Valaciclovir}

Valaciclovir is a prodrug of aciclovir which may also be used to treat herpes zoster ophthalmicus. The recommended oral dose is $1 \mathrm{~g}$ three times a day for seven days.

\section{Trifluorothymidine}

Trifluorothymidine (TFT) is a thymidine analogue that inhibits the enzyme thymidine synthetase. Therefore, it has a different mechanism of action to acyclovir. This implies that TFT should be effective in cases of aciclovir-resistant herpetic keratitis. Unfortunately, it is no longer available in South Africa, despite still being widely used in the USA and Canada.

\section{Antifungal ophthalmic preparations}

Fungal keratitis is a sight-threatening condition that requires specialist management. A fungal aetiology should always be considered in any keratitis that results from an injury involving organic matter, especially if the injury leads to indolent ulceration. An interesting fact about fungal keratitis is that currently there is only one available commercial preparation for its treatment. The drug, natamycin, is derived from the bacterium, Streptomyces natalensis, which was first discovered in the former Natal province of South Africa and is now used worldwide as a first-choice agent in many cases of fungal keratitis. All other ophthalmic topical antifungal agents need to be specially prepared by a pharmacist and should be reserved for specialist use.

\section{Antiparasitic ophthalmic preparations}

Many parasites, both microscopic and macroscopic, may infect different parts of the eye. Examples include Acanthamoeba keratitis, Microsporidia keratitis, Pneumocystis jiroveci choroiditis and Toxoplasma gondii retinitis. Infestation by Onchocerca volvulus, a helminth, causes onchocerciasis or river blindness, which commonly occurs in neighbouring countries such as Zimbabwe. It is also sporadically seen in South Africa. Treatment comprises a single $150 \mu \mathrm{g} / \mathrm{kg}$ dose of oral ivermectin. The dose may be repeated after six months if the patient is still symptomatic. All of these infections require specialist management, although fairly simple drugs sometimes play an important role in their treatment. Propamidine isethionate is sometimes used to treat superficial bacterial ocular infections, but also plays an important role in the treatment of Acanthamoeba keratitis, in combination with other agents such as $0.02 \%$ chlorhexidine gluconate solution.

\section{Miscellaneous ophthalmic preparations}

\section{Povidone iodine}

Povidone iodine $5 \%$, an organic iodine antiseptic agent, is widely used in the preoperative cleansing of the periocular skin and ocular surface. It has brought about a dramatic reduction in the incidence of postoperative 
endophthalmitis. ${ }^{10}$ It has been shown that a $2.5 \%$ solution used on the conjunctivae of newborns was more effective than both silver nitrate and erythromycin in preventing ophthalmia neonatorum, ${ }^{11}$ but not quite as effective as $1 \%$ tetracycline ointment. ${ }^{12}$ However, the latter is not available in South Africa. Povidone iodine $2 \%$ possesses antiv iral properties and has proved to be effective in relieving the symptoms of $77 \%$ of patients who were treated for epidemic keratoconjunctivitis ("pink eye") within a week. ${ }^{13}$ Therefore, povidone iodine is an important anti-infective ophthalmic preparation. It plays both a prophylactic and a therapeutic role.

\section{Antibiotic-steroid combinations}

Many of the topical antibacterial agents are also available in combination with a topical corticosteroid. Examples include Tobradex $^{\circledR}$ (tobramycin plus dexamethasone), Spersadex Comp $^{\circledR}$ (chloramphenicol plus dexamethasone) and Betnesol $\mathrm{N}^{\circledR}$ (betamethasone plus neomycin). These drugs are predominantly used before and after eye operations to decrease inflammation and prevent infection in the operated eye. Their use is not recommended in the treatment of blepharitis, conjunctivitis or keratitis of unknown origin since the corticosteroid component will exacerbate any undiagnosed herpetic infection. Therefore, the use of combination therapy is not encouraged in general practice. ${ }^{2}$

\section{Conclusion}

The number of topical anti-infective ophthalmic preparations that are available commercially is relatively small. The majority of these agents are intended for use against bacterial infections which are relatively easy to diagnose and treat. First-line drugs, such as tobramycin, chloramphenicol, ciprofloxacin and ofloxacin, are currently still very effective in curing most superficial bacterial ocular infections. The third-generation fluoroquinolones, gatifloxacin and moxifloxacin, should preferably be reserved for cases that do not respond to first-line agents to minimise the development of resistance to these drugs. Oral antibacterial drugs may also be required to prevent and treat certain ophthalmic conditions.
Far fewer commercial options exist for the treatment of viral, fungal and parasitic infections. Both topical and oral aciclovir play an important role in the treatment of ocular herpetic infections. To prevent iatrogenic exacerbation, these infections need to be actively excluded before considering the use of a topical preparation that contains a corticosteroid. Fungal and parasitic infections often mimic bacterial infections and are then only diagnosed when they do not respond to antibacterial therapy. These conditions are notoriously difficult to treat and should rather be referred for specialist management.

\section{References}

1. Rhee DJ, Colby KA, Rapuano CJ, et al. Ophthalmic drug guide. $1^{\text {st }}$ ed. New York: Springer; 2007.

2. Rossiter $\mathrm{D}$, Blockman $\mathrm{M}$, Barnes $\mathrm{Kl}$, et al, editors. South African medicines formulary. $10^{\text {th }}$ ed. Cape Town: Health and Medical; 2012.

3. Jackson WB, Low DE, Dattani D, et al. Treatment of acute bacterial conjunctivitis: $1 \%$ fusidic acid viscous drops vs. $0.3 \%$ tobramycin drops. Can J Ophthalmol. 2002;37(4):228-237.

4. Kibel MA. Silver nitrate and the eyes of the newborn: a centennial. S Afr Med J. 1981;60(26):979-980.

5. Laporte J, Vidal X, Ballarin E, et al. Possible association between ocular chloramphenicol and aplastic anaemia: the absolute risk is very low. $\mathrm{Br} \mathrm{J}$ Clin Pharmacol. 1998;46(2):181-184.

6. Croswell J. Ocular prophylaxis for gonococcal ophthalmia neonatorum. Am Fam Physician. 2012;85(2):197-198.

7. Oliphant CM, Green GM. Quinolones: a comprehensive review. Am Fam Physician. 2002;65(3):455-465.

8. Kanski JJ. Clinical ophthalmology: a systematic approach. $6^{\text {th }}$ ed. Philadelphia: Elsevier, 2007; p. 221-225.

9. Athmanathan S, Garg P, Rao GN. Ophthalmic antiviral chemotherapy: an overview. Indian J Ophthalmol. 1997;45(4):203-210.

10. Speaker MG, Menikoff JA. Prophylaxis of endophthalmitis with topical povidone-iodine.

11. Ophthalmology. 1991;98(2):1769-1775.

12. Isenberg SJ, Apt L, Yoshimori A, et al. Povidone-lodine for ophthalmia neonatorum prophylaxis. Am J Ophthalmol. 1994;118(6):701-706.

13. David M, Rumelt S,Weintraub Z. Efficacy comparison between povidone iodine $2.5 \%$ and tetracycline $1 \%$ in prevention of ophthalmia neonatorum. Ophthalmology 2011;118(7):1454-1458.

14. Trinavarat A, Atchaneeyasakul LO. Treatment of epidemic keratoconjunctivitis with 2\% povidone-iodine: a pilot study. J Ocul Pharmacol Ther. 2012 Feb;28(1):53-58

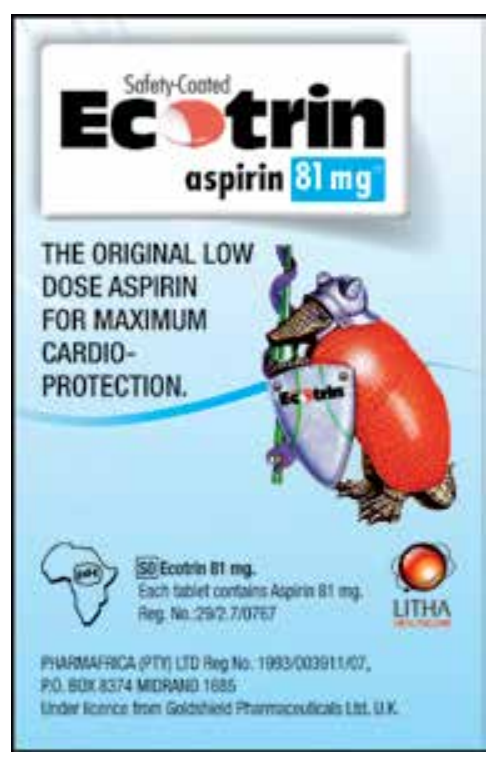

